\title{
Industry updates from the field of stem cell research and regenerative medicine in September 2020
}

\author{
Dusko llic*,1(i) \& Mirjana Liovic ${ }^{2}$ (iD) \\ ${ }^{1}$ Stem Cell Laboratories, Guy's Assisted Conception Unit, Department of Women \& Children's Health, Faculty of Life Sciences \& \\ Medicine, King's College London, London, SE1 9RT, UK \\ ${ }^{2}$ Medical Center for Molecular Biology, Faculty of Medicine, University of Ljubljana, 1000 Ljubljana, Slovenia \\ *Author for correspondence: dusko.ilic@kcl.ac.uk
}

Latest developments in the field of stem cell research and regenerative medicine compiled from publicly available information and press releases from nonacademic institutions in September 2020.

First draft submitted: 20 October 2020; Accepted for publication: 3 November 2020; Published online: 17 November 2020

Keywords: industry $\bullet$ regenerative medicine $\bullet$ stem cells

\section{Business development}

Collaboration agreement: Kiadis \& AWRI

Kiadis Pharma (Amsterdam, the Netherlands; www.kiadis.com), a cellular therapy company, has announced a collaboration with the Abigail Wexner Research Institute (AWRI; OH, USA; www.nationwidechildrens.org) at Nationwide Children's Hospital to develop Kiadis-NK cells (K-NK cells) as a postexposure pre-emptive therapy for COVID-19 [1]. The US FDA-approved AWRI's investigational new drug application (IND) for a study in an adult population with off-the-shelf natural killer (NK) cells produced with Kiadis' proprietary Universal Donor and PM21 technologies. Kiadis and AWRI are developing the plan for initiation of the clinical study. Kiadis has exclusively licensed from AWRI intellectual property related to NK cells for treatment of microbial infections, including SARS-CoV-2. The Company has recently initiated the preclinical and clinical development of its K-NK-ID101 COVID-19 program and is expecting to receive US government funding for this program.

\section{Collaboration agreement: Kiadis \& GCRBC}

Kiadis Pharma, a clinical-stage biopharmaceutical company developing innovative NK-cell-based medicines for the treatment of life-threatening diseases and Gulf Coast Regional Blood Center (GCRBC; TX, USA; www.gi veblood.org), a primary supplier of blood components to more than 170 hospitals and healthcare facilities, have announced a collaboration under which GCRBC will supply universal donor starting material for the manufacture of Kiadis' off-the-shelf K-NK cell therapies in the US [2]. Kiadis' proprietary off-the-shelf K-NK cell platform is based on NK cells from unique universal donors. This collaboration will provide Kiadis with an ongoing supply of starting material needed for clinical supply and research and development. The Company is developing multiple K-NK programs utilizing universal donor starting material.

\section{Collaboration agreement: Kite \& HiFiBio}

Kite Pharma (CA, USA; www.kitepharma.com), a Gilead Company (CA, USA; www.gilead.com), and HiFiBiO Therapeutics (MA, USA; www.hifibio.com) have announced the companies have entered into a 2 year research collaboration and license agreement in acute myeloid leukemia (AML) [3]. Through this collaboration, $\mathrm{HiFiBiO}$ will use its proprietary technology platforms to identify novel AML targets and anti-AML specific antibodies for Kite's use in cell therapies. Under the terms of the agreement, $\mathrm{HiFiBiO}$ will receive an upfront payment and will be eligible for additional payments based on the achievement of certain research milestones. Kite will have an exclusive option to opt in on any targets discovered through the collaboration, for which $\mathrm{HiFiBiO}$ will receive an additional 
payment and will be eligible for additional development, regulatory and commercial milestone payments, as well as royalty payments.

\section{Collaboration agreement: lineage cell therapeutics \& AgeX}

Lineage Cell Therapeutics (CA, USA; www.lineagecell.com), cellular therapy company, ES Cell International (CA, USA; https://esibio.com/), and AgeX Therapeutics (CA, USA; www.agexinc.com), have announced the broadening of their collaborative relationship with regard to ES Cell's human embryonic stem cell lines [4]. ESI cell lines are current Good Manufacturing Practice-compatible, registered with the NIH. Neither party made or received any cash payments regarding this arrangement. Lineage acquired exclusivity for ESI Cell Lines in spinal cord injury and certain oncology indications. AgeX secured independence to license out ESI Cell Lines as part of its collaboration and licensing model.

\section{Collaboration agreement: Vor \& Arbor Biotechnologies}

Vor Biopharma (MA, USA; www.vorbiopharma.com), an oncology company pioneering engineered hematopoietic stem cells (HSC) for the treatment of cancer, and Arbor Biotechnologies (MA, USA; https://arbor.bio), an earlystage life sciences company, have announced an agreement to use Arbor's gene editing technologies to engineer HSC, toward the goal of developing therapies for the treatment of blood cancers, such as AML [5].

\section{Licensing agreement: Chimeric \& City of Hope}

Chimeric Therapeutics (Australia; www.chimerictherapeutics.com), a drug development company focused on novel chimeric antigen receptor (CAR-T) cell therapies for solid tumors, has entered a licensing agreement with US cancer research and treatment center, City of Hope (CA, USA; www.cityofhope.org), for the intellectual property relating to its pioneering chlorotoxin (CLTX) CAR-T cell therapy [6,7]. CARs commonly incorporate a monoclonal antibody sequence in their targeting domain, enabling CAR-T cells to recognize antigens and kill tumor cells. In contrast, the CLTX CAR uses a synthetic 36-amino acid peptide sequence first isolated from death stalker scorpion venom and now engineered to serve as the CAR recognition domain. The therapy, which uses a peptide derived from scorpion toxin to direct $\mathrm{T}$ cells to target glioblastoma, is being used in a Phase I clinical trial at City of Hope. The first patient in the trial recently started treatment. Under the licensing agreement, Chimeric has acquired the exclusive worldwide rights to develop and commercialize City of Hope's CLTX CAR-T cells, as well as further develop the therapy for other cancers.

\section{Merger agreement: Orgenesis \& Koligo}

Orgenesis (MD, USA; www.orgenesis.com) and Koligo Therapeutics (IN, USA; www.koligo.net) have announced that the two companies have entered into a definitive merger agreement, subject to final closing conditions, with expected completion before year end [8]. Koligo has successfully launched its first commercial product, KYSLECEL, autologous stromal and vascular fraction cells derived from adipose tissue, and plans to commence a Phase II trial of KT-PC-301 for COVID-19-related acute respiratory distress syndrome. Koligo's development stage technology utilizes 3D bioprinting and vascularization. Following closing of the transaction, Orgenesis plans to accelerate the commercial scale-up of KYSLECEL throughout the USA and, subject to regulatory and logistical considerations, in international markets as well. After closing of the transaction, and subject to FDA review and clearance of the Company's IND application. Under the terms of the merger agreement, Orgenesis will acquire all the outstanding stock of Koligo from its shareholders. The agreed consideration terms are an aggregate of $\$ 15$ million in shares of Orgenesis' common stock valued at $\$ 7.00$ per share which shall be issued to Koligo's accredited investors (with certain nonaccredited investors to be paid solely in cash) and an assumption of $\$ 1.3$ million in Koligo's liabilities, estimated to be substantially all of Koligo's liabilities.

\section{Launching new products \& services Curi Bio}

Curi Bio (WA, USA; www.curibio.com), a developer of human induced pluripotent stem cell (hiPSC)-based platforms for drug discovery, has launched the ComboMat ${ }^{\top \mathrm{M}}$ platform, a proprietary hiPSC-derived cardiomyocyte (hiPSC-CMs) maturation technology [9]. Curi's ComboMat platform leverages a combination of cues - including a proprietary microRNA cocktail - to drive the functional development and maturation of cardiomyocytes with applications in drug discovery, disease modeling and safety and efficacy screening. By providing drug developers 
more mature and clinically relevant human cardiac stem cells for use in preclinical drug development, Curi aims to help pharmaceutical partners develop safer and more effective therapeutics in less time, at lower cost. Curi will make the ComboMat platform available to pharmaceutical customers through service contracts and partnerships with applications in drug discovery, disease modeling and safety and efficacy screening. Several days later, the company also launched the Mantarray ${ }^{\top M}$ platform for human-relevant 3D engineered muscle tissue analysis [10].

\section{FUJIFILM}

FUJIFILM Irvine Scientific (CA, USA; www.irvinesci.com) announced that it has become the exclusive, worldwide distributor of Cellnest ${ }^{\circledR}$, a recombinant peptide attachment substrate that provides optimal adhesion and proliferation of stem cells in chemically defined, animal component-free conditions [11]. Cellnest is compatible with any adherent cell type that binds to the Arg-Gly-Asp (RGD) domain, an amino acid sequence within the extracellular matrix protein fibronectin that mediates cell attachment. Cellnest was designed and manufactured by FUJIFILM Corporation.

\section{OrganaBio}

OrganaBio (FL, USA; www.organabio.com) has launched the MesenPAC ${ }^{T M}$ and ImmunoPAC ${ }^{T M}$ product lines, designed to support translational researchers in their quest to rapidly develop next generation cell-based therapies [12]. These products provide a unique and timely solution to critical supply chain issues that are hindering the regenerative medicine, cell therapy, gene therapy and immunotherapy arenas. MesenPAC, mesenchymal stem/stromal cells (MSCs), and ImmunoPAC, NK cells, are derived from full-term placenta, umbilical cord tissue and umbilical cord blood obtained from consented, noncompensated donors under IRB approved protocols.

\section{Takeda}

Takeda Pharmaceutical (Japan; www.takeda.com) has announced the expansion of its cell therapy manufacturing capabilities with the opening of a new $2230 \mathrm{~m}^{2}$ (24,000 square-foot) R\&D cell therapy manufacturing facility at its R\&D headquarters in Boston [13]. The facility provides end-to-end research and development capabilities and will accelerate Takeda's efforts to develop next-generation cell therapies, initially focused on oncology with potential to expand into other therapeutic areas.

\section{Clinical trials \\ Pluripotent stem cells \\ Cynata}

Cynata Therapeutics (Australia; www.cynata.com), a cellular therapy company, has published the data from the Phase I clinical trial of CYP-001 in patients with graft versus host disease (GvHD) [14,15]. The published trial results detail the world's first clinical trial of an allogeneic hiPSC-derived product. CYP-001, Cynata's lead iPSC-derived MSC product candidate broke ground by being the first MSC therapy to be produced at scale without the limitation of multiple donors through Cynata's novel Cymerus ${ }^{T M}$ technology (https://clinicaltrials.gov; ID: NCT02923375).

In the clinical trial, 15 patients with steroid-resistance acute GvHD received two infusions each of Cymerus MSCs. The trial was conducted at seven clinical centers in the UK and Australia. The first eight participants were enrolled in cohort A and received two infusions of CYP-001 at a dose of 1 million cells per kg of body weight (cells $/ \mathrm{kg}$ ), up to a maximum dose of 100 million cells. Seven participants in cohort B received two infusions of CYP-001 at a dose of 2 million cells $/ \mathrm{kg}$, up to a maximum dose of 200 million cells. There was one week between the two CYP-001 infusions in each participant.

The trial's primary objective was to assess the safety and tolerability of CYP-001, while the secondary objective was to evaluate the efficacy of two infusions of CYP-001 in adults with steroid-resistant GvHD. The primary evaluation period concluded 100 days after the first dose in each participant. Efficacy was assessed on the basis of response to treatment (as determined by change in GvHD grade) and overall survival at 28 and 100 days after the administration of the first dose. After the completion of the primary evaluation period, participants entered a longer-term, noninterventional follow-up period, which will continue for up to 2 years after the initial dose.

Key results of the clinical trial were as follows:

- Overall response rate by day 100 was $87 \%$ (13/15 patients showed an improvement in GvHD severity by at least one grade compared with baseline); 
- Complete response rate by day 100 was 53\% (GvHD signs and symptoms completely resolved in 8/15 patients);

- Overall survival at day 100 was $87 \%$;

- No treatment-related serious adverse events or safety concerns were identified.

\section{Hematopoietic stem cells Orchard}

Orchard Therapeutics (UK; www.orchard-tx.com) has announced additional interim data from an ongoing proofof-concept clinical trial evaluating the safety and efficacy of OTL-203, an investigational ex vivo autologous HSC gene therapy in development for the treatment of mucopolysaccharidosis Type I (MPS-I) at the San Raffaele Telethon Institute for gene therapy (SR-Tiget) in Milan, Italy [16].

MPS-I is a rare, inherited neurometabolic disease caused by a deficiency of the alpha-L-iduronidase (IDUA) lysosomal enzyme, which is required to break down glycosaminoglycans (GAGs). The accumulation of GAGs across multiple organ systems results in symptoms including neurocognitive impairment, skeletal deformity, loss of vision and hearing and cardiovascular and pulmonary complications. MPS-I occurs at an overall estimated frequency of one in every 100,000 live births. There are three subtypes of MPS-I; approximately $60 \%$ of children born with MPS-I have the most severe subtype, called Hurler syndrome and rarely live past the age of ten when untreated. OTL-203 is an investigational ex vivo autologous HSC gene therapy being studied for the treatment of MPS-I. Orchard was granted an exclusive worldwide license to intellectual property rights to research, develop, manufacture and commercialize the gene therapy program for the treatment of MPS-I developed by the San Raffaele Telethon Institute for gene therapy in Milan (Italy; https://research.hsr.it/en/institutes/san-raffaele-telethon-institute-for-ge ne-therapy.html).

Eight patients with the severe Hurler subtype of MPS-I had been treated with OTL-203 in the ongoing proofof-concept study, which completed enrollment in December 2019. As of July 2020, all patients had been followed for a minimum of 6 months, with the longest follow-up extending out to 24 months. Treatment with OTL-203 was generally well-tolerated with a safety profile consistent with the selected conditioning regimen. Consistent with previous analyses, treatment across all eight patients continued to demonstrate:

- Rapid hematologic reconstitution, with neutrophil and platelet engraftment within 21 days following treatment;

- Biological efficacy established by sustained supranormal alpha-L-iduronidase (IDUA) enzyme expression in peripheral blood for all patients within 3 months postgene therapy and up to 18 months in the first treated patient;

- Metabolic correction as measured by reduction in GAG levels in urine achieved in all patients by 6 months postgene therapy, with sustained correction out to 18 months in the first treated patient.

Further results for the first two treated patients demonstrated:

- Rapid metabolic correction of GAG levels in the cerebral spinal fluid, reflecting restoration of IDUA enzyme expression in the CNS;

- Improved motor function and acquisition of cognitive and language skills;

- Stabilization or improvement in white matter abnormalities, cervical canal stenosis, kyphosis and vertebra deformity, as measured by brain and spine MRI scores;

- Continued growth progression above the 50th percentile of normal;

- Improved range of motion (an indicator of joint stiffness).

The company also announced that EMA has granted Priority Medicines (PRIME) designation to OTL-203 [17].

\section{Regulations, approvals \& acquisitions Green light \\ AlloVir}

AlloVir (MA, USA; www.allovir.com), a cellular therapy company, announced that the FDA has cleared the IND for ALVR109, an allogeneic, off-the-shelf virus-specific T-cell therapy candidate designed to target SARS-CoV-2, the virus that causes the severe and life-threatening viral disease, COVID-19 [18]. ALVR109 is being developed to arrest the progression of COVID-19 by eradicating SARS-CoV-2 virus-infected cells. 


\section{Atara Biotherapeutics}

Atara Biotherapeutics (CA, USA; www.atarabio.com), a pioneer in T-cell immunotherapy leveraging its novel allogeneic Epstein-Barr Virus T-cell platform to develop treatments for patients with severe diseases including solid tumors, hematologic cancers and autoimmune disease, has announced that the FDA has accepted the IND application providing clearance to initiate an open-label, single-arm Phase I clinical study of ATA2271, the Company's next-generation autologous CAR-T therapy targeting mesothelin under development in collaboration with Memorial Sloan Kettering Cancer Center (MSKCC; NY, USA; www.mskcc.com), for the treatment of advanced mesothelioma [19].

In collaboration with MSKCC, Atara is developing ATA2271, a next-generation autologous mesothelin-targeted CAR-T using novel 1XX CAR signaling and programmed death-1 dominant negative receptor (PD1DNR) checkpoint inhibition technologies (M28z1XX PD1DNR CAR-T cells). This technology is supported by the safety and antitumor efficacy that was exhibited in prior studies evaluating a mesothelin-directed CAR utilizing a CD28 costimulatory signaling domain. This autologous mesothelin-targeted construct (using M28z CAR-T cells) combined with PD-1 antibody is being studied in two ongoing MSK Phase I studies in patients with malignant pleural disease and mesothelioma, non-small-cell lung cancer and breast cancer (NCT02414269 and NCT02792114).

\section{Athenex}

Athenex (NY, USA; www.athenex.com) has announced that the FDA has allowed its IND application for TCRTESOA2, an autologous T-cell receptor (TCR)-T-cell therapy targeting solid tumors that are NY-ESO-1 positive in

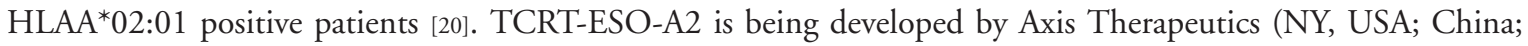
www.axistherapeutics.com), a joint venture between Athenex and Xiangxue Life Sciences, a subsidiary of Xiangxue Pharmaceutical (China; www.xphcn.com). TCRT-ESO-A2 is similar to T-cell receptor Affinity Enhancing Specific T-cell therapy (TAEST) 16001, an autologous cell-based therapy being developed simultaneously by XLifeSc for clinical application in China in that both therapies express the same affinity-enhanced TCR. TAEST technology platform that provides high binding affinity of TCRs while reducing their off-target toxicity.

\section{AVROBIO}

AVROBIO (MA, USA; www.avrobio.com), a clinical-stage gene therapy company, has announced that the European Commission (EC) has granted orphan drug designation for AVR-RD-02, the company's investigational lentiviral gene therapy for the treatment of Gaucher disease [21]. AVR-RD-02 consists of the patient's own hematopoietic stem cells, genetically modified to express functional glucocerebrosidase (GCase), the enzyme that is deficient in Gaucher disease. AVR-RD-02 has also received orphan drug designation from the FDA. AVROBIO recently dosed the first patient in the GuardOne Phase I/II clinical trial to evaluate the safety and efficacy of AVR-RD-02 (NCT04145037).

Gaucher disease is a rare, inherited lysosomal storage disorder characterized by the toxic accumulation of glucosylceramide (GlcCer) and glucosylsphingosine (GlcSph) in macrophages. Macrophages bloated with these fatty substances are called Gaucher cells which amass primarily in the spleen, liver and bone marrow. This results in a variety of potential symptoms, including grossly enlarged liver and spleen, bone issues, fatigue, low hemoglobin levels and platelet counts and an adjusted lifetime relative risk of developing Parkinson's disease that may be more than 20-times greater than the general population. Even on enzyme replacement therapy - the current standard of care - people with Gaucher disease Type 1 typically have a shortened life expectancy and may experience debilitating symptoms that significantly reduce their quality of life. An estimated one in 44,000 people are diagnosed with Gaucher disease.

Baylx

Baylx (CA, USA; www.baylxinc.com), a clinical-stage stem cell biopharmaceutical company focused on developing living stem cell products to address unmet medical needs, announced that the FDA has cleared Baylx's IND application for BXU001, a fresh, nonfrozen human umbilical cord tissue MSC product in patients with COVID19 [22]. This Phase I/IIa clinical trial of Baylx will recruit approximately 30 COVID-19 patients with moderate to severe acute respiratory distress syndrome, an acute hypoxemic respiratory failure with high mortality rate. 


\section{bluebird}

bluebird bio (MA, USA; www.bluebirdbio.com) has announced that its investigational gene therapy for sickle cell disease (SCD), LentiGlobin ${ }^{T M}$ (bb1111), was granted eligibility to the Priority Medicines (PRIME) program by the EMA [23]. The EMA's PRIME initiative provides enhanced support and increased interaction to companies, with the goal of optimizing development plans and speeding regulatory evaluations to potentially bring innovative medicines to patients more quickly. To be accepted for PRIME, a therapy must demonstrate potential to benefit patients with unmet medical need through early clinical data. LentiGlobin for SCD was designed to add functional copies of a modified form of the $\beta$-globin gene $\left(\beta^{\mathrm{A}-\mathrm{T} 87 \mathrm{Q}}\right)$ globin gene) into a patient's own HSC. Once patients have the $\beta^{\mathrm{A}-\mathrm{T} 87 \mathrm{Q}}$-globin gene, their red blood cells can produce anti-sickling hemoglobin, $\mathrm{Hb} \mathrm{A}^{\mathrm{A}-\mathrm{T} 87 \mathrm{Q}}$, which decreases the proportion of $\mathrm{HbS}$, with the goal of reducing sickled red blood cells, hemolysis and other complications. bluebird bio's clinical development program for LentiGlobin for SCD includes the completed Phase I/II HGB205 study (NCT02151526), the ongoing Phase I/II HGB-206 study (NCT02140554), and the ongoing Phase III HGB-210 study (NCT04293185). bluebird bio is conducting a long-term safety and efficacy follow-up study (LTF-303) for people who have participated in bluebird biosponsored clinical studies of betibeglogene autotemcel for $\beta$-thalassemia or LentiGlobin for SCD (NCT02633943).

\section{Mustang Bio}

Mustang Bio (MA, USA; www.mustangbio.com), a clinical-stage cell and gene therapy company, has announced that the FDA has granted Orphan drug designation to MB-207, Mustang's ex vivo lentiviral gene therapy for the treatment of patients with X-linked severe combined immunodeficiency (XSCID), also known as bubble boy disease, who have been previously treated with a hematopoietic stem cell transplantation and for whom retreatment is indicated [24]. The FDA previously granted rare pediatric disease, Orphan drug and regenerative medicine advanced therapy designations to MB-107 for the treatment of XSCID in newly diagnosed infants. Additionally, the EMA granted advanced therapy medicinal product classification to MB-107 [25].

XSCID is a rare genetic disorder that occurs in approximately one per 225,000 births. It is characterized by the absence or lack of function of key immune cells, resulting in a severely compromised immune system and death by 1 year of age if untreated. Patients with XSCID have no T cells or NK cells. Although their B cells are normal in number, they are not functional. The specific genetic disorder that causes XSCID is a mutation in the gene coding for the common gamma chain $(\gamma \mathrm{c})$, a protein that is shared by the receptors for at least six interleukins. These interleukins and their receptors are critical for the development and differentiation of immune cells. The gene coding for $\gamma c$ is known as IL-2 receptor gamma, or IL2RG. Because IL2RG is located on the X-chromosome, XSCID is inherited in an X-linked recessive pattern, resulting in almost all patients being male.

\section{Vericel}

Vericel Corporation (MA, USA; www.vcel.com) has announced that the FDA has accepted for filing the recently submitted Biologics License Application for NexoBrid ${ }^{\circledR}$ (concentrate of proteolytic enzymes enriched in bromelain) for eschar removal (debridement) in adults with deep partial-thickness and/or full-thickness thermal burns [26]. The FDA assigned a Prescription Drug User Fee Act target date of 29 June 2021.

\section{Capital market \& finances \\ Chimeric}

Chimeric Therapeutics (Australia; www.chimerictherapeutics.com), a drug development company focused on novel CAR-T cell therapies for solid tumors, has raised US\$ 3.0 (A \$4.3) million via convertible note through Baker Young (Australia; www.bakeryoung.com.au), as it progresses a Phase I clinical trial in glioblastoma for the Company's CLTX CAR-T technology [27]. Following the completion of the convertible note round, Bell Potter Securities (Australia; https://bellpotter.com.au) has joined with Baker Young Limited as the corporate advisors to the company.

\section{Kiadis}

Kiadis Pharma (the Netherlands; www.kiadis.com), a cellular therapy company, has received US\$ 9.5 million in funding from the Advanced Regenerative Manufacturing Institute's (ARMI; NH, USA; www.armiusa.org) BioFabUSA program, in partnership with the United States Department of Defense, to fund Kiadis' K-NK-ID101 program [28]. The funds from ARMI BioFabUSA provides funding for Kiadis' research on K-NK-ID101's activity 
and mode of action in COVID-19 treatment, including the recently announced Dutch research program. In addition, the funds will support a new Company-sponsored Phase I/IIa clinical trial to evaluate K-NK-ID101 in COVID-19 and the scale up of current Good Manufacturing Practice manufacturing. Additionally, Kiadis will collaborate with ARMI BioFabUSA to establish large-scale manufacturing capacity for K-NK-ID101 in the US that can support the industrialization of K-NK cell therapy. ARMI BioFabUSA received funding from the United States Department of Defense to advance large-scale manufacturing of engineered tissues and tissue-related technologies, including cell therapy.

\section{Lineage Cell Therapeutics}

Lineage Cell Therapeutics (CA, USA; www.lineagecell.com), cellular therapy company, has been awarded a new research \& development grant for 2020 of up to 1.95 million Israeli New Shekels (approximately US\$ 573,000) from the Israel Innovation Authority (IIA; Israel; https://innovationisrael.org.il/en/) [29]. The grant, which is one of the first under the IIA's bio-convergence initiative, provides funding for the development of a novel bio-retinal patch for the treatment of retinal diseases by Lineage's subsidiary, Cell Cure Neurosciences (Israel; www.cellcureneurosciences.com), in partnership with Precise Bio (NC, USA \& Israel; www.precise-bio.com).

\section{Stemson}

Stemson Therapeutics (CA, USA; www.stemsontx.com) has announced a US $\$ 7.5$ million seed financing led by Allergan Aesthetics (www.allerganaesthetics.com), an AbbVie Company (IL, USA; www.abbvie.com), and impact investor Fortunis Capital (UK; www.fortuniscapital.co.uk) to advance development of Stemson's therapeutic solution to cure hair loss [30]. Stemson aims to restore human hair growth with a novel approach using the patient's own cells to generate new hair follicles.

\section{Financial \& competing interests disclosure}

Dusko llic has received an honorarium from Future Science Group for the contribution of this work. The authors have no other relevant affiliations or financial involvement with any organization or entity with a financial interest in or financial conflict with the subject matter or materials discussed in the manuscript apart from those disclosed.

No writing assistance was utilized in the production of this manuscript.

\section{References}

1. Kiadis Pharma. Kiadis announces US FDA approval of the Abigail Wexner Research Institute at Nationwide Children's Hospital's IND for a COVID-19 clinical trial with off-the-shelf K-NK cells using Kiadis' proprietary platforms (2020). https://ir.kiadis.com/node/7581/pdf

2. Kiadis Pharma. Kiadis and Gulf Coast Regional Blood Center announce collaboration to provide universal donor material for K-NK cell therapy programs (2020). https://ir.kiadis.com/node/7606/pdf

3. Kite Pharma. Kite and HiFiBio therapeutics partner to discover novel targets and antibodies against acute myeloid leukemia (2020). http://www.kitepharma.com/news/press-releases/2020/9/kite-and-hifibio-therapeutics-partner-to-discover-novel-targets-and-antibodi es-against-acute-myeloid-leukemia

4. Lineage Cell Therapeutics. Lineage Cell Therapeutics and AgeX Therapeutics announce expansion of agreement related to ESI clinical-grade pluripotent stem cell lines for therapeutic purposes (2020). https://investor.lineagecell.com/node/20671/pdf

5. Vor Biopharma. Vor Biopharma and arbor biotechnologies to collaborate on engineered hematopoietic stem-cell therapies (2020). http://www.vorbio.com/vor-biopharma-and-arbor-biotechnologies-to-collaborate-on-engineered-hematopoietic-stem-cell-therapies/

6. Chimeric Therapeutics. Chimeric Therapeutics enters licensing agreement with City of Hope to develop pioneering Phase I chlorotoxin CAR-T cell therapy (2020). https://www.chimerictherapeutics.com/wp-content/uploads/2020/09/Chimeric-Therapeutics-enters-licensi ng-agreement-with-City-of-Hope-to-develop-CLTX-CAR-T-cell-therapy-.pdf

7. Wang D, Starr R, Chang WC et al. Chlorotoxin-directed CAR T cells for specific and effective targeting of glioblastoma. Sci. Transl. Med. 12(533), eaaw2672 (2020).

8. Orgenesis. Orgenesis announces agreement to acquire koligo therapeutics, a leader in personalized cell therapies (2020). https://ir.orgenesis.com/press-releases/detail/165/orgenesis-announces-agreement-to-acquire-koligo

9. Business Wire. Curi Bio launches ComboMat platform for iPSC-based drug discovery (2020). http://www.businesswire.com/news/home/20200910005359/en/Curi-Bio-Launches-ComboMat-Platform-iPSC-based-Drug

10. BusinessWire. Curi Bio announces Mantarray ${ }^{\top M}$ platform for analysis of $3 \mathrm{D}$ engineered muscle tissues for discovery of new therapeutics (2020). http://www.businesswire.com/news/home/20200915005905/en/Curi-Bio-Announces-MantarrayTM-Platform-Analysis-3D 
11. FUJIFILM Irvine Scientific. FUJIFILM irvine scientific to exclusively distribute chemically defined substrate for stem cell culture (2020). http://www.irvinesci.com/uploads/uploaded-press-releases/Fujifilm_Irvine_Cellnest_Launch_PR_FISI_15Sep20.pdf

12. OrganaBio. OrganaBio launches portfolio of novel perinatal tissue-derived products to spur rapid development of cell and gene therapies (2020). https://www.organabio.com/wp-content/uploads/2020/09/Perinatal-Cell-Portfolio-Sept2020.pdf

13. Takeda Pharmaceuticals. Takeda opens new R\&D cell therapy manufacturing facility to support expansion of next-generation clinical programs (2020). http://www.takeda.com/newsroom/newsreleases/2020/takeda-opens-new-rd-cell-therapy-manufacturing-facility-to --support-expansion-of-next-generation-clinical-programs/

14. Cynata Therapeutics. Results of Cynata's world-first clinical trial of iPSC-derived CYP-001 in GvHD accepted for publication in Nature Medicine (2020). http://files.cynata.com/617/200914CynataClinicalTrialResultsPublishedinNatureMedicine.pdf

15. Bloor AJC, Patel A, Griffin JE et al. Production, safety and efficacy of iPSC-derived mesenchymal stromal cells in acute steroid-resistant graft versus host disease: a Phase I, multicentre, open label, dose-escalation study. Nat. Med. doi:10.1038/s41591-020-1050-x (2020) (Epub ahead of print)

16. Orchard Therapeutics. Orchard Therapeutics Announces additional interim results from Proof-of-Concept Study of OTL-203 for MPS-I (2020). https://ir.orchard-tx.com/node/7681/pdf

17. Orchard Therapeutics. Orchard Therapeutics receives EMA PRIME designation for OTL-203 for the treatment of MPS-I (2020). https://ir.orchard-tx.com/node/7711/pdf

18. AlloVir. AlloVir announces the FDA clearance of investigational new drug application (IND) for ALVR109 for the treatment of high-risk COVID-19 patients (2020). https://ir.allovir.com/node/7061/pdf

19. Atara Biotherapeutics. Atara Biotherapeutics announces FDA clearance of IND for ATA2271, a next-generation autologous mesothelin-targeted CAR T with novel PD1DNR and 1XX Technologies combined to treat solid tumors (2020). https://investors.atarabio.com/news-events/press-releases/detail/205/atara-biotherapeutics-announces-fda-clearance-of-ind-for

20. Athenex. Athenex announces FDA allowance of IND application for TCRT-ESO-A2, a TCR-T cell therapy (2020). https://ir.athenex.com/news-releases/news-release-details/athenex-announces-fda-allowance-ind-application-tcrt-eso-a2-tcr\#

21. AVROBIO. AVROBIO receives orphan drug designation from the European Medicines Agency for AVR-RD-02, an investigational gene therapy for gaucher disease (2020).

https://investors.avrobio.com/news-releases/news-release-details/avrobio-receives-orphan-drug-designation-european-medicines

22. Baylx. BAYLX, INC. Announced clearance of COVID-19 IND from US FDA for cord mesenchymal stem cells (2020). http://www.baylxinc.com/post/baylx-inc-announced-clearance-of-covid-19-ind-from-us-fda-for-cord-mesenchymal-stem-cells

23. bluebird. bluebird bio's LentiGlobin ${ }^{\top \mathrm{M}}$ for sickle cell disease gene therapy (bb1111) granted priority medicines (PRIME) designation by European Medicines Agency (2020). http://investor.bluebirdbio.com/news-releases/news-release-details/bluebird-bios-lentiglobintm-sickle-cell-disease-gene-therapy

24. Mustang Bio. Mustang Bio announces orphan drug designation for MB-207 for the treatment of X-linked severe combined immunodeficiency ("XSCID") in previously transplanted patients (2020).

https://ir.mustangbio.com/News/news-details/2020/Mustang-Bio-Announces-Orphan-Drug-Designation-for-MB-207-for-the-Trea tment-of-X-linked-Severe-Combined-Immunodeficiency-XSCID-in-Previously-Transplanted-Patients/default.aspx

25. Mustang Bio. Mustang Bio announces orphan drug designation for MB-107 for the treatment of X-linked severe combined immunodeficiency in newly diagnosed infants (2020).

https://ir.mustangbio.com/News/news-details/2020/Mustang-Bio-Announces-Orphan-Drug-Designation-for-MB-107-for-the-Trea tment-of-X-linked-Severe-Combined-Immunodeficiency-in-Newly-Diagnosed-Infants/default.aspx

26. Vericel. Vericel announces FDA acceptance of biologics license application for nexobrid for the treatment of severe thermal burns (2020). https://investors.vcel.com/node/18846/pdf

27. Chimeric Therapeutics. Chimeric Therapeutics completes A $\$ 4.3 \mathrm{M}$ seed capital raising for ground-breaking Phase I CAR-T asset (2020). http://www.chimerictherapeutics.com/wp-content/uploads/2020/09/Chimeric-completes-4.3m-seed-capital-raising.pdf

28. Kiadis Pharma. Kiadis receives $\$ 9.5$ million from the Advanced Regenerative Manufacturing Institute’s BioFabUSA Program to fund Kiadis K-NK cell COVID-19 therapy (2020). https://ir.kiadis.com/node/7601/pdf

29. Lineage Cell Therapeutics. Lineage Cell Therapeutics awarded grant from Israel Innovation Authority for development of a bio-retinal patch for treatment of retinal diseases in partnership with Precise Bio (2020). https://investor.lineagecell.com/node/20661/pdf

30. Stemson Therapeutics. Stemson Secures $\$ 7.5 \mathrm{M}$ seed financing to advance hair regeneration therapy for hair loss (2020). https://stemsontx.com/stemson-secures-7-5m-seed-financing-to-advance-hair-regeneration-therapy-for-hair-loss/ 